\title{
O. Shawki
}

\section{Laparoscopy and the anterior abdominal wall: a guide to vascular mapping for safe entry}

Published online: 24 February 2004

(C) Springer-Verlag Berlin / Heidelberg 2004

\begin{abstract}
Laparoscopy is an invaluable diagnostic and therapeutic tool. Unfortunately, the introduction of trocars into the abdominal cavity is not without certain specific inherent dangers, such as injury to the vasculature of the anterior abdominal wall. This article assesses the normal vasculature of the anterior abdominal wall during laparoscopy as well as various methods, such as pneumoperitoneum pressure and patient positioning, for improving the safety margins for the insertion of umbilical as well lateral trocars.
\end{abstract}

Keywords Laparoscopy - Anatomy of abdominal wall vessels · Pneumoperitoneum · Pressure - Safe entry

\section{Introduction}

The development of laproscopic surgery marshalled in a new era in terms of both diagnostics and therapeutics, often eliminating the need for open abdominal surgery. However, as with all developing techniques, as usage increases so does the number of reported associated complications. Although relatively few, minor complications are reported in as many as $5.1 \%$ of laparoscopic procedures and major complications in upwards of $2.3 \%$ [1]. Complications related to laparoscopic procedures include ureteric injury $[2,3]$ as well as damage to other internal organs, including the bowels, bladder and uterus [4]. Vascular trauma has also been associated with the placement of needles and trocars, where the incidence of injury to the vessels of the anterior abdominal wall [5] occurs with greater frequency than injury to deep vessels,

\footnotetext{
O. Shawki

Department of Gynecology,

Cairo University,

Cairo, Egypt

O. Shawki (

Al Ebtesama Hospital,

10 Aboul Magd Amer Street, Heliopolis, 11341 Cairo, Egypt

e-mail: romeo@menanet.net

Fax: +202-3651881
}

such as the aorta and iliac vessels [6]. Despite the use of relatively standardized techniques and increasing experience of the surgeons, complications related to the insertion of trocars still occur [7].

The anatomical relations of the anterior abdominal wall may be variable and different under pneumoperitoneum. The insufflation of the abdomen will stretch the anterior abdominal wall and change the standard anatomical landmarks and measurements.

The aim of this study was to assess the anatomical relation of the anterior abdominal wall to underlying structures and the effects of manipulation of various parameters during trocar insertion under different pneumoperitoneum pressures.

\section{Materials and methods}

Thirty women undergoing diagnostic laparoscopy for the investigation of infertility consented to participate in the study. The study was conducted during the period from March through November 2001. The women were selected based on having no previous history of abdominal surgery. The mean duration of infertility for the group was 6.1 years, the mean age 27.6 years, and the mean body weight $68.3 \mathrm{~kg}$. For the purposes of the study, the patients were divided into three groups. In group A, direct trocar insertion was used in the absence of prior insufflation, while in group B insufflation was used to attain an intraabdominal pressure of between $15 \mathrm{mmHg}$ to $20 \mathrm{mmHg}$. Finally, group $\mathrm{C}$ comprised those patients in whom the intraabdominal pressure was raised to $25 \mathrm{mmHg}$ prior to trocar insertion.

In each patient a preliminary primary puncture was made $5 \mathrm{~cm}$ cranial to the symphasis pubis in midline, with a $5 \mathrm{~mm}$ trocar through which a $5 \mathrm{~mm}$ laproscope was introduced in order to monitor the introduction of the subumbilical $10 \mathrm{~mm}$ primary trocar. During the introduction of the main subumbilical $10 \mathrm{~mm}$ trocar, the distance between the anterior abdominal wall and the great vessels and viscera was visually observed and assessed. Particular attention was paid to the indentation effects of trocar thrust. In addition transillumination techniques were used to assess the location of the inferior epigastric, superficial epigastric and superficial circumflex arteries, as well as the lateral margins of the rectus muscle, in relation to the symphasis pubis, the umbilicus and the abdominal mid-line. The results were evaluated to determine the safest possible entry technique in terms of the following: (1) site of 
puncture, (2) intra-abdominal pressure adjustment, (3) patient position, (4) trocar type and (5) direction of trocar thrust.

\section{Results}

Assessment of the vasculature of the anterior abdominal wall revealed the following: at $5 \mathrm{~cm}$ above the symphysis pubis, the inferior and superficial epigastric arteries lay $5.3 \pm 1 \mathrm{~cm}$ and $5.8 \pm 2 \mathrm{~cm}$ from the linea alba, respectively (Fig. 1). Near the level of the umbilicus, the lateral edge of the rectus sheath was located $7.3 \pm 1.7 \mathrm{~cm}$ from the midline. At the same level, the superficial epigastric and circumflex iliac vessels were positioned at $4.6 \pm 1.8 \mathrm{~cm}$ and $11.2 \pm 1.6 \mathrm{~cm}$ from the linea alba, respectively. The umbilicus was commonly located cranial to the bifurcation of the aorta; pneumoperitoneum caused the umbilicus to move even more cranially, thus increasing the distance between the umbilicus and the aortic bifurcation. Positioning the patient in Trendelenburg's position tends to alter the position of the lower part of the aorta, bringing it

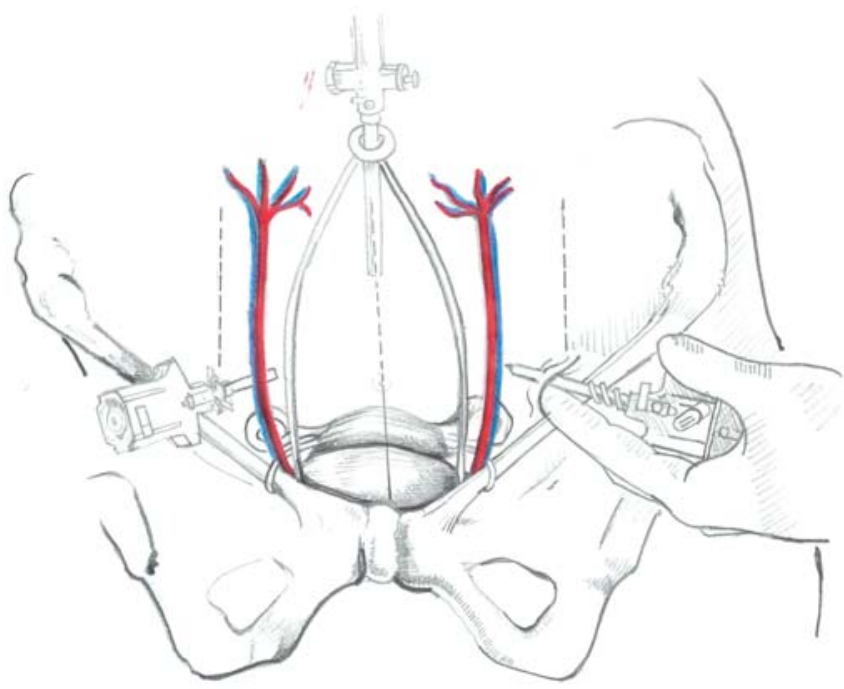

Fig. 1 Anatomy of anterior abdominal wall vessels and relations to secondary punctures points

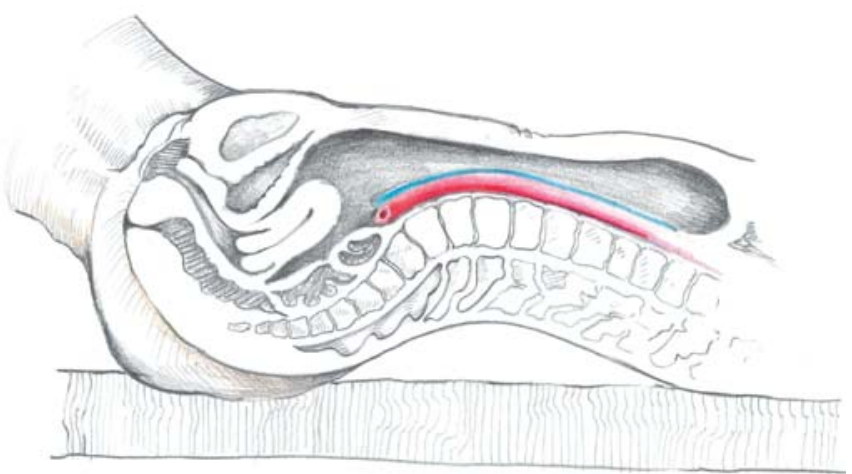

Fig. 2 Relation of anterior abdominal wall to great vessels under general anaesthesia prior to pneumoperitoneum

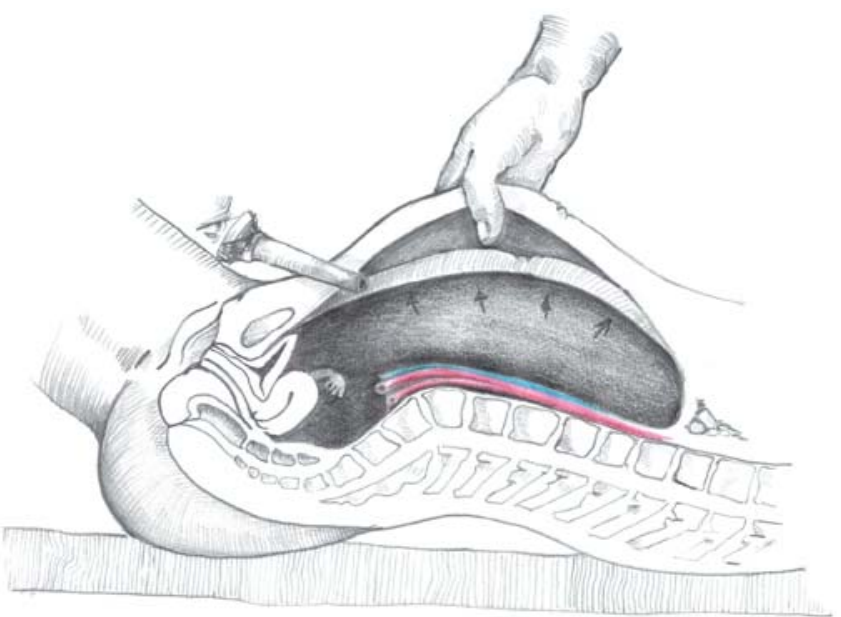

Fig. 3 The effect of manual elevation of the anterior abdominal wall showing distance created by manual lift

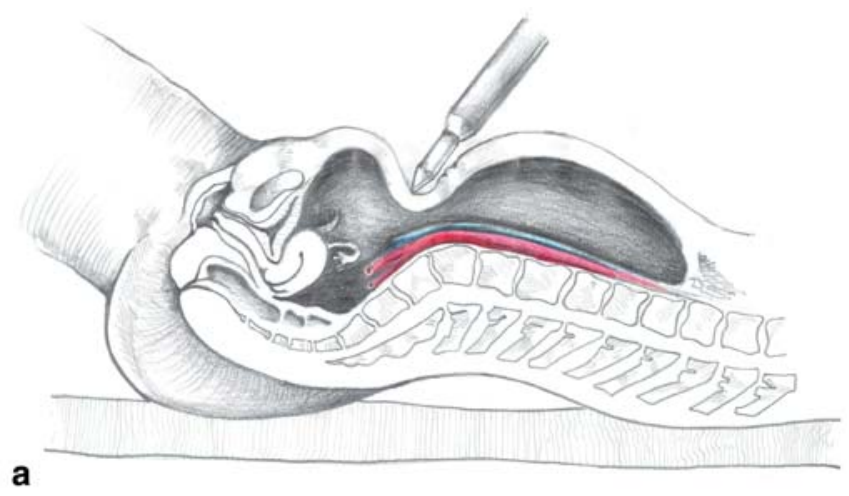

a

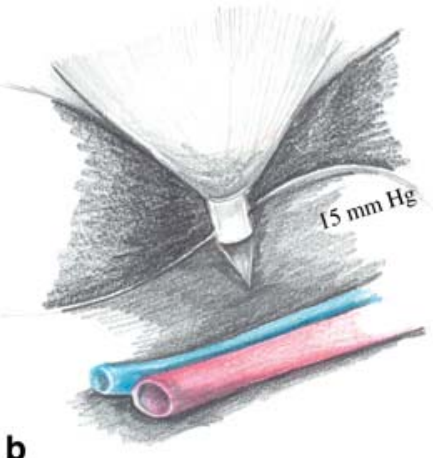

Fig. 4 Thrust effect of umbilical trocar insertion under standard pneumoperitoneum $(15 \mathrm{mmHg})$ and proximity to posterior abdominal wall great vessels during low pressure thrust

closer to the umbilicus. Under GA, the anterior abdominal wall lies nearly directly over the great vessels (Fig. 2). By using manual elevation, the distance between the anterior abdominal wall and the retroperitoneal vessels can be increased to $7 \pm 2 \mathrm{~cm}$ in the average-weight patient (Fig. 3) and marginally less so in the obese. Insufflation also increases the wall-to-wall distance, therefore increasing the safety margin. 

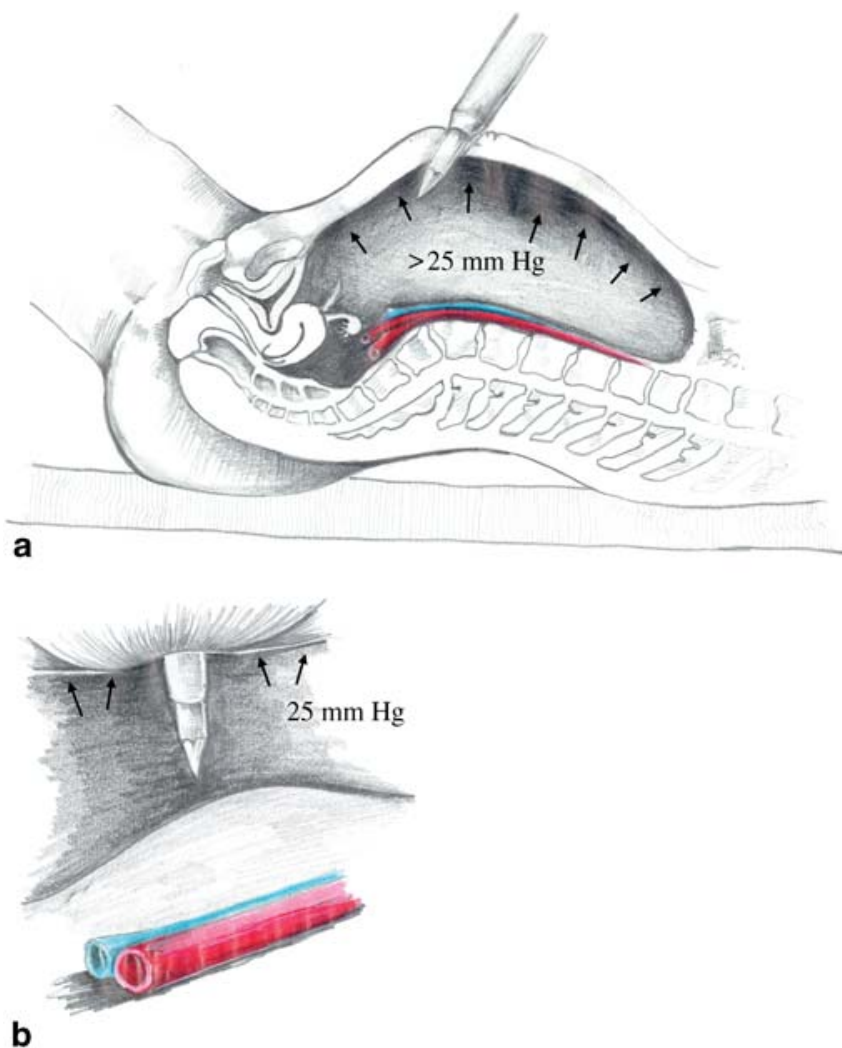

Fig. 5 Thrust effect of umbilical trocar insertion under high pneumoperitoneum $(20-25 \mathrm{mmHg})$ and proximity to posterior abdominal wall great vessels during high pressure thrust

Pneumoperitoneum of $15 \mathrm{mmHg}$ to $20 \mathrm{mmHg}$ resulted in more force being required to penetrate the abdomen. This forced insertion resulted in sagging and indentation of the anterior abdominal wall to such an extent as to bring the anterior wall half the distance closer to the retroperitoneal structures, thus negating the effect of insufflation (Fig. 4). Following insufflation to $25 \mathrm{mmHg}$, trocar penetration required less force and the retroperitoneal structures remained more than $10 \mathrm{~cm}$ from the point of insertion (Fig. 5).

It is quite conceivable that penetration of a tightly inflated balloon is much easier than of a low pressure one that will dent and yield under force (Fig. 6).

\section{Discussion}

The positions of the anterior abdominal vessels described in this study are similar to those described by other authors $[8,9]$.
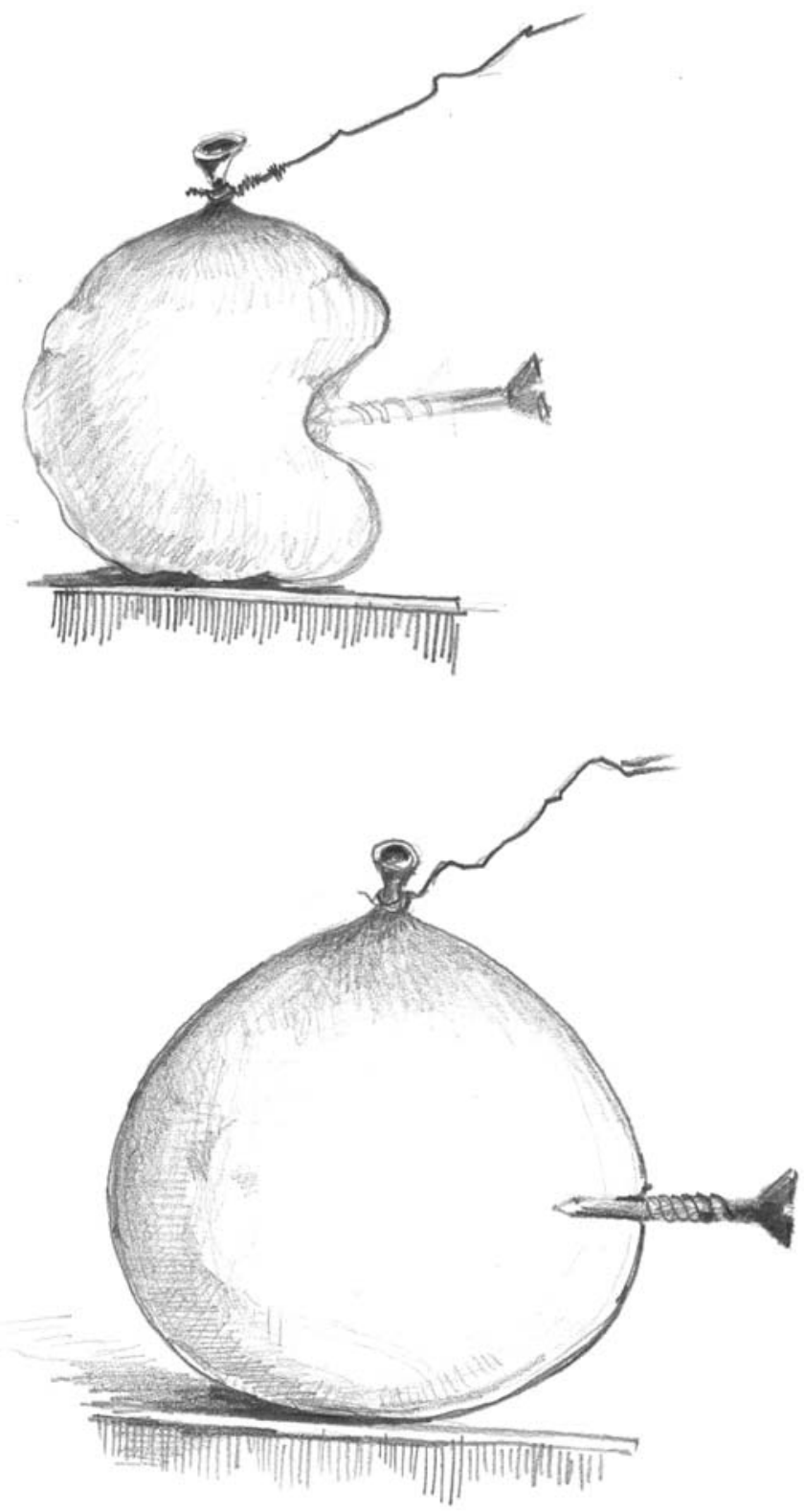

Fig. 6 Diagrammatic representation for puncturing balloon under low and high pressure

Additionally, our study is the first to report direct observation of primary trocar entry and to observe the thrust effect on anterior abdominal wall approximation to posterior structures. The results obtained in this study and that of Hurd et al. [8] are compared in Table 1. The significance of these results and their similarity to those obtained in other studies means that it is now possible to
Table 1 Positions of anterior abdominal vessels from the midline (cm; Fig. 1). The figures in brackets indicate the values obtained in the current study

\begin{tabular}{lllc}
\hline Distance from symphysis & $3 \mathrm{~cm}$ & $5 \mathrm{~cm}$ & $16 \mathrm{~cm}$ \\
\hline Inf. epigastric a & $5.6 \pm 1$ & $5.2 \pm 1.2(5.3 \pm 1)$ & 4.6 \\
Sup. epigastric a & $5.5 \pm 2$ & $5.2 \pm 1.8(5.8 \pm 2)$ & $4.6 \pm 1.4(4.6 \pm 1.8)$ \\
Sup. circumflex a & $8.5 \pm 1$ & $9.5 \pm 1.6$ & $10.7 \pm 1.7(11.2 \pm 1.6)$ \\
Lat. margin of rectus m & & & $7.6 \pm 1.5(7.3 \pm 1.7)$ \\
\hline
\end{tabular}


map out the most probable locations of the vessels of the anterior abdominal wall, with confidence, to avoid them during laparoscopy. Although the positions of these vessels do not usually present problems in diagnostic laparoscopy, where midline placement of trocars is the norm, the placement of lateral trocars does increase the risk of vascular injury. The placement of lateral trocars at a distance of $3 \mathrm{~cm}$ above the symphysis pubis further increases the risk of vascular injury because the distance between the more laterally located superficial circumflex artery and the inferior and superficial epigastric vessels is often less than $2 \mathrm{~cm}$. However, this risk can be lessened by placing trocars medial to the positions of the vessels. Lateral trocar placement at $5 \mathrm{~cm}$ above the symphysis pubis is all around a safer alternative because the gap between the above mentioned vessels is larger $(>4 \mathrm{~cm})$. Near the umbilicus, it is prudent to place trocars at $8 \mathrm{~cm}$ from the midline in the gap between the epigastric and superficial circumflex iliac vessels. Finally, in terms of vasculature, it is essential to consider the issue of normal human variation and that the guidelines given here represent the most common arrangement of the vessels of the anterior abdominal wall. Variations and anomalies do occur, albeit infrequently $[9,10]$.

The umbilicus tends to be used as a landmark from which measurement is made for the positioning of trocars. Furthermore, the position of the umbilicus is used as an indicator of the position of the aortic bifurcation to avoid retroperitoneal vessel injury [11]. The bifurcation is usually located at or cranial to the umbilicus, thus increasing the risk of damage to the vessel during trocar insertion. Pneumoperitoneum always results in the cranial movement of the umbilicus. Where insufflation is used and the pressure created is not sufficiently high, the risk of injury is greater because of the greater force required for insertion and the fact that the indentation of the abdominal wall halves the distance between the skin and the retroperitoneal vessels. Obesity also plays a role in increasing the risk of retroperitoneal vessel injury where it is suggested that trocar insertion occurs at a $90^{\circ}$ angle to the skin to avoid preperitoneal placement [12]. Injury to retroperitoneal vessels can, however, be avoided by taking the precautions recommended by Loffer and Pent [13].

\section{Conclusion}

The avoidance of vascular injury during placement of lateral trocars is of paramount importance. The following recommendations are made: (1) In accordance with Hurd et al. [8] and our observation, in the absence of laparoscopic anatomical landmarks to guide placement, lateral trocars should be inserted at least $5 \mathrm{~cm}$ above the pubic symphysis and $8 \mathrm{~cm}$ from the linea alba. (2) Umbilical inscision should be strictly intraumbilical and not infra- umbilical. The placement of the patient in Trendelenburg's position should only be carried out after trocar insertion to avoid risk of aorta proximity. (3) The use of insufflation to achieve a pneumoperitoneum with a pressure of $25 \mathrm{mmHg}$ allows for a safer and easier entry technique. (4) Safe trocar entry in the absence of pneumoperitoneum can only be achieved in thin patients with lax abdomens where direct manual abdominal lift can be ensured, preferably using smaller caliber trocars, like the 5 or $7 \mathrm{~mm}$ ones. (5) The trocar found to offer the safest use is the short disposable palm trocar, because it requires only half the force for insertion. (6) Finally, an in-depth knowledge of the anatomy of the vasculature of the anterior abdominal wall is one of the cornerstones for safe trocar placement.

Acknowledgements The author acknowledges the support of Prof. P. Richards, head of the Department of Anatomy, Medical Faculty, University of Pretoria, South Africa, in fulfilling this research and her continuous collaboration and efforts.

\section{References}

1. Kane MG, Krejis GJ (1984) Complications of diagnostic laparoscopy in Dallas: a 7-year prospective study. Gastrointest Endosc 30:237-240

2. Cheng YS (1976) Ureteral injury resulting from laparoscopic fulguration of endometriotic implant. Am J Obstet Gynecol $1045-1046$

3. Grainger DA, Soderstrom RM, Schiff SF, et al (1990) Ureteral injuries at laparoscopy: insights into diagnosis, management, and prevention. Obstet Gynecol 75:839-843

4. Yuzpe AA (1990) Pneumoperitoneum needle and trocar injuries in laparoscopy. J Repro Med 35:485-490

5. Hurd WW, Pear ML, Delancey JOL, et al (1993) Laparoscopic injury of abdominal wall blood vessels: a report of three cases. Obstet Gynecol 82:673-676

6. Baadsgaard SE, Bille S, Egeblad K (1989) Major vascular injury during gynecologic laparoscopy. Acta Obstet Gynecol Scan 68:283-285

7. Hurd WW, Hurtcau (1997) Practical manual of laparoscopy and hysteroscopy. Springer, New York Berlin Heidelberg

8. Hurd WW, Bude RO, Delancey JOL, et al (1994) The location of abdominal wall blood vessels in relationship to abdominal landmarks apparent at laparoscopy. Am J Obstet Gynecol 171:642-646

9. Jakubowicz M, Czarniawska-Grzesinska M (1996) Variability in origin and topography of the inferior epigastric and obturator arteries. Folia Morph 55:121-126

10. Bilgic S, Sahin B (1997) Rare arterial variation: a common trunk from the external iliac artery for the obturator, inferior epigastric and profunda femoris arteries. Surg Rad Anat 19:4547

11. Hurd WW, Bude RO, Delancey JOL, et al (1992) The relationship of the umbilicus to the aortic bifurcation: implications for laparoscopic technique. Obstet Gynecol 80:48-51

12. Hurd WW, Bude RO, Delancey JOL, et al (1991) Abdominal wall characterization with magnetic resonance imaging and computed tomography: the effect of obesity on the laparoscopic approach. J Reprod Med 36:473-476

13. Loffer FD, Pent D (1974) An alternative technique in penetrating the abdomen for laparoscopy. J Reprod Med 13:37-40 